\title{
USING GENERALIZED STOCHASTIC METHOD TO EVALUATE PROBABILITY OF CONFLICT IN CONTROLLED AIR TRAFFIC
}

\author{
V. Babak, V. Kharchenko, V. Vasylyev \\ National Aviation University, 1 Kosmonavta Komarov Ave., 03058, Kiev, Ukraine.E-mail:v_vasylyev@ukr.net \\ Received 2701 2007, accepted 11062007
}

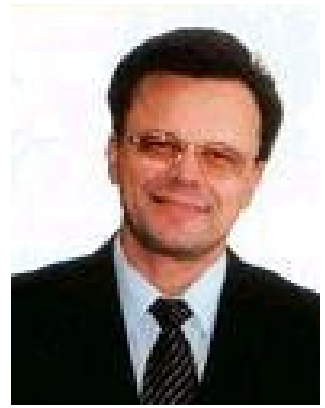

Vitaly BABAK, Prof Dr Habil

Education: Kiev Polytechnic Institute

Rector of National Aviation University, Director of the Institute of Information and Diagnostic Systems, 1995 DSc (Eng.), Member of Academy of Sciences, Ukraine

Areas of research: diagnostics of technological processes, laser and computer modelling and manufacturing.

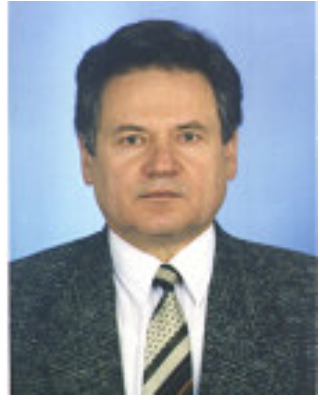

Volodymyr KHARCHENKO, Dr

Education: Kiev Institute of Civil Aviation Engineers

Vice-rector of National Aviation University for scientific work, Head of Aeronavigation Systems Department, 1994 — DSc (Eng.)

Areas of research: theory of situational analysis and regulation in sociotechnical systems.

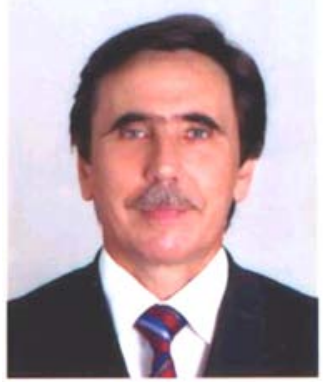

Volodymyr VASYLYEV, Prof Dr Habil

Education: Kiev Institute of Civil Aviation Engineers

Professor of Aeronavigation Systems Department at the National Aviation University, 2006 - DSc (Eng.)

Areas of research: systems theory, identification and prediction, decision support systems and their application to the problems in air navigation and air traffic management.

Abstract. The introduction of the new concepts of air traffic management (ATM) and transition from centralized to decentralized air traffic control (ATC) with the change of traditional ATM to Cooperative ATM sets new tasks and opens new capabilities for air traffic safety systems. This paper is devoted to the problem of evaluating the probability of aircraft collision under the condition of Cooperative ATM, when the necessary information is available to the subjects involved in the decision-making process. The generalized stochastic conflict probability evaluation method is developed. This method is based on the generalized conflict probability equation for evaluation of potential conflict probability and aircraft collision probability that is derived by taking into account stochastic nature and time correlation of deviation from planned flight trajectory in controlled air traffic. This equation is described as a multi-dimensional parabolic partial differential equation using a differential (infinitesimal) operator of the multi-dimensional stochastic process of relative aircraft movement. The common procedure for the prediction of conflict probability is given, and the practical application of the generalized method presented is shown. All equational coefficients of a differential operator for a practical solution of a parabolic partial differential equation are derived. For some conditions, the numerical solution of the conflict probability equation is obtained and illustrated graphically.

Keywords: air traffic management, conflict detection, conflict probability, collision risk, stochastic method. 


\section{Introduction}

In connection with the introduction of new concepts of air traffic management (ATM) and transition from centralized to decentralized air traffic control (ATC) and with the change from traditional ATM to Cooperative ATM, the problem of air traffic safety is nowadays actively discussed by many researchers $[6,9,5]$.

Cooperative ATM is a concept of air traffic management that enhances the productivity and safety of air traffic services by optimising the involvement of controllers, aircrew and airline operators through integrated data communications and improved forms of surveillance and automation $[9,5]$.

But in conditions of predicted growth of air traffic intensity, change of dynamics of relative aircraft movement and reduction of separation standard the role of the systems of detecting and preventing the dangerous aircraft approaching should be highly increased.

Nowadays the stochastic (probabilistic) methods of detecting and estimating conflict situations are considered the most promising [4]. All known stochastic methods can be divided into two groups. One group is based on predicting the stochastic process of aircraft deviation from a planned trajectory and subsequently analysing the predicted relative position of the aircraft [2, 3]. These methods are complicated mathematically and a simplifying transformation is needed for their realization

Another group of methods is based on the prediction of the aircraft position uncertainty area with subsequent analysis of their dangerous approach [7, 1]. The methods of this group do not give a sufficiently reliable result when the aircraft are approaching closer than 5 nautical miles. So these methods cannot estimate the risk of collision.

This paper is devoted to the problem of evaluating the probability of aircraft collision under the condition of Cooperative ATM, when the necessary information is available to the subjects involved in the decision-making process. In particular, the ATC system should have access to navigation data that are contained in on-board flight management systems (FMS). The new characteristics of Cooperative ATM enable to develop intent-based conflict detection and resolution methods that use the active flight plan (intent) as a basis for trajectory prediction and conflict detection.

In this paper, the generalized stochastic conflict probability evaluation method belonging to the first group mentioned above is developed. This method is based on the generalized conflict probability equation for evaluating potential conflict probability and aircraft collision probability that is derived with regard to the stochastic nature and time correlation of deviation from planned flight trajectory under controlled air traffic.

\section{Mathematical model of an aircraft motion under control}

The basis of any method to evaluate conflict probability is a mathematical model of the aircraft flight process.
Consider a mathematical model of aircraft motion taking into account the control factor. An aircraft moves under control along a prescribed route. Altitude, flight direction, and velocity along the route are stabilized as a result of control.

The key idea is to describe the deviation from planned trajectory by the Ornstein-Uhlenbeck (OU) random process, and derive the relative motion of two aircraft as multidimensional diffusion process.

Consider a local horizontal coordinate system $x O y$, with an axis $O x$ along a prescribed route, where

$x(t)$ is a longitudinal coordinate at the moment $t$;

$y(t)$ is a lateral coordinate;

$\Delta y(t)=y(t)$ is deviation from the route line;

$v_{x}(t)$ is aircraft velocity;

$v_{0}$ is prescribed (planned) velocity;

$u_{x}(t)=v_{x}(0)-v_{0}$ is deviation from the prescribed velocity (Fig 1).

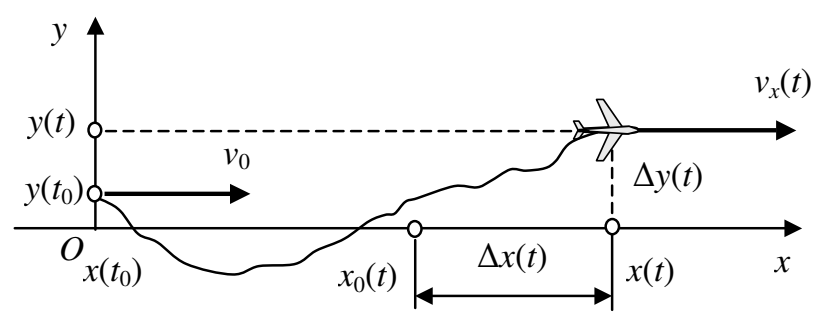

Fig 1. Aircraft movement in a local coordinate system

The model of deviation from the route line as the OU process is described by the equation

$$
d y(t)=-\alpha_{y} y(t) d t+\sigma_{y} d W_{y}(t)
$$

where $y(0)$ is known by observations, $\alpha_{y}$ and $\sigma_{y}$ are known positive scalars, and $\left\{W_{y}(t)\right\}$ is a standard Wiener process.

Note that the OU process is not divergent and allows one to take into account the stabilizing factor of trajectory parameters.

The longitudinal coordinate $x(t)$ is described by the equation

$$
d x(t)=\left(v_{0}+u_{x}(t)\right) d t, \quad x(0)=0
$$

where $v_{0}$ is a prescribed velocity, and $u_{x}(t)$ is deviation from the prescribed velocity, which is described by $\mathrm{OU}$ process as well,

$$
d u_{x}(t)=-\alpha_{x} u_{x}(t) d t+\sigma_{x} d W_{x}(t)
$$

where $u_{x}(0)$ is known by observation, $\alpha_{x}$ and $\sigma_{x}$ are positive scalars, and $\left\{W_{x}(t)\right\}$ is another standard Wiener process, independent of $\left\{W_{y}(t)\right\}$. 


\section{Mathematical model of aircraft relative motion}

Now consider the relative motion of two aircraft at the same altitude. A state vector in the corresponding local coordinates characterizes each aircraft

$$
\mathbf{z}(t)=\left[x(t), \quad y(t), u_{x}(t)\right]^{\mathrm{T}} .
$$

Based on equations (1)-(3) we have

$$
d \mathbf{z}(t)=\left[v_{0} \mathbf{e}+\Lambda\left[\begin{array}{c}
y(t) \\
u_{x}(t)
\end{array}\right]\right] d t+\mathbf{S} d \mathbf{W}(t)
$$

$$
\begin{gathered}
\text { where } \quad \mathbf{e}=[1,0,0]^{T}, \quad \boldsymbol{\Lambda}=\left[\begin{array}{cc}
0 & 1 \\
-\alpha_{y} & 0 \\
0 & -\alpha_{x}
\end{array}\right] \\
\mathbf{W}(t)=\left[\begin{array}{l}
W_{x}(t) \\
W_{y}(t)
\end{array}\right], \mathbf{S}=\left[\begin{array}{cc}
0 & 0 \\
0 & \sigma_{y} \\
\sigma_{x} & 0
\end{array}\right]
\end{gathered}
$$

Consider the common horizontal coordinate system $x O y$. The motion of each aircraft is described in the local coordinate system $x_{j} O_{j} y_{j}, j=1,2$. Let $\mathbf{e}_{1}, \mathbf{e}_{2}$ be unit vectors for the first aircraft and $\mathbf{f}_{1}, \mathbf{f}_{2}$ be unit vectors for the second one, as shown in the picture.

Let $\mathbf{r}(0)=\mathbf{r}_{0}=\mathbf{r}_{20}-\mathbf{r}_{10}$ be a vector corresponding to the initial position between two aircraft.

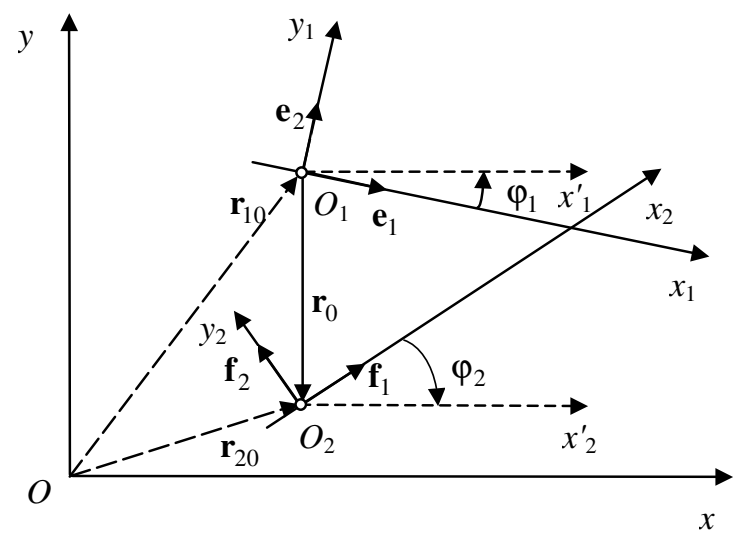

Fig 2. Relative motion of two aircraft

Then the vector $\mathbf{r}(t)$ of the relative position of the aircraft equals

$$
\mathbf{r}(t)=\left(\mathbf{r}_{20}-\mathbf{r}_{10}\right)+x_{2}(t) \mathbf{f}_{1}+y_{2}(t) \mathbf{f}_{2}-x_{1}(t) \mathbf{e}_{1}-y_{1}(t) \mathbf{e}_{2} \cdot(6)
$$

Transform the state vector (4) of each aircraft from local coordinates to common coordinates:

$$
\mathbf{R}(t)=\left[\begin{array}{ll}
\mathbf{U} & 0 \\
0 & 1
\end{array}\right] \mathbf{z}(t)
$$

where $\mathbf{U}_{j}=\left[\begin{array}{cc}\cos \varphi_{j} & -\sin \varphi_{j} \\ \sin \varphi_{j} & \cos \varphi_{j}\end{array}\right]$ is the turn matrix.

Then equation (5) implies the equation

$$
d \mathbf{R}(t)=(\mathbf{h}+\mathbf{M R}(t)) d t+\mathbf{\Sigma} d \mathbf{W}(t)
$$

where

$$
\begin{gathered}
\mathbf{h}=v_{0}\left[\begin{array}{c}
\mathbf{U}\left[\begin{array}{l}
1 \\
0
\end{array}\right] \\
0
\end{array}\right], \quad \boldsymbol{\Sigma}=\left[\begin{array}{cc}
\mathbf{U} & 0 \\
0 & \mathbf{U}
\end{array}\right] \mathbf{S} ; \\
\mathbf{M}=\left[\begin{array}{ll}
\mathbf{U} & 0 \\
0 & 1
\end{array}\right] \mathbf{\Lambda} \mathbf{P}_{23}\left[\begin{array}{cc}
\mathbf{U}^{\mathrm{T}} & 0 \\
0 & 1
\end{array}\right] .
\end{gathered}
$$

The matrix $\mathbf{P}_{23}$ is a projector

$$
\mathbf{P}_{23}=\left[\begin{array}{lll}
0 & 1 & 0 \\
0 & 0 & 1
\end{array}\right]
$$

such that $\mathbf{P}_{23}\left[r_{1}, r_{2}, r_{3}\right]^{T}=\left[r_{2}, r_{3}\right]^{T}$.

Consider the compound vector for two aircraft

$$
\mathbf{X}(t)=\left[\begin{array}{l}
\mathbf{R}_{1}(t) \\
\mathbf{R}_{2}(t)
\end{array}\right]
$$

and using (7)-(8) derive the diffusion equation

$$
d \mathbf{X}(t)=\left[\begin{array}{c}
\mathbf{h}_{1}+\mathbf{M}_{1} \mathbf{R}_{1}(t) \\
\mathbf{h}_{2}+\mathbf{M}_{2} \mathbf{R}_{2}(t)
\end{array}\right] d t+\left[\begin{array}{cc}
\boldsymbol{\Sigma}_{1} & 0 \\
0 & \boldsymbol{\Sigma}_{2}
\end{array}\right] d\left[\begin{array}{c}
W_{x 1}(t) \\
W_{y 1}(t) \\
W_{x 2}(t) \\
W_{y 2}(t)
\end{array}\right],
$$

or

$$
d \mathbf{X}(t)=\mathbf{F}(t) d t+\mathbf{L}(t) d \mathbf{W}^{\prime}(t)
$$

that describes in general form the deviation processes for two aircraft simultaneously.

Here $\left\{W_{x j}(t), W_{y j}(t), \quad j=1,2\right\}, \quad$ are mutually independent Wiener processes.

The initial state $\mathbf{X}(0)$ is given by the observations.

\section{Conflict probability evaluation}

\subsection{The infinitesimal operator}

Consider the equation for estimating conflict probability using the differential (infinitesimal) operator of the corresponding diffusion process [8]. This operator enables one to determine correspondence between the diffusion process and its probability performances.

The structure of the infinitesimal operator in general form was determined in paper [4]. To obtain the operator for process (9), it is necessary to find matrix $\mathbf{F}$, for which according to equation (8) we have for each aircraft 


$$
\begin{gathered}
\mathbf{h}+\mathbf{M R}= \\
=\left[\begin{array}{c}
v_{0} \cos \varphi-\alpha_{y} \sin ^{2} \varphi x+\alpha_{y} \sin \varphi \cos \varphi y+\cos \varphi u_{x} \\
v_{0} \sin \varphi+\alpha_{y} \sin \varphi \cos \varphi x-\alpha_{y} \cos ^{2} \varphi y+\sin \varphi u_{x} \\
-\alpha_{x} u_{x}
\end{array}\right]
\end{gathered}
$$

and find the diffusion matrix that is equal

$$
\mathbf{L L}^{\mathrm{T}}=\left[\begin{array}{cc}
\boldsymbol{\Sigma}_{1} \boldsymbol{\Sigma}_{1}^{\mathrm{T}} & 0 \\
0 & \boldsymbol{\Sigma}_{2} \boldsymbol{\Sigma}_{2}^{\mathrm{T}}
\end{array}\right]
$$

for which we have

$$
\Sigma \Sigma^{T}=\left[\begin{array}{ccc}
\sigma_{y}^{2} \sin ^{2} \varphi & -\sigma_{y}^{2} \sin \varphi \cos \varphi & 0 \\
-\sigma_{y}^{2} \sin \varphi \cos \varphi & \sigma_{y}^{2} \cos ^{2} \varphi & 0 \\
0 & 0 & \sigma_{x}^{2}
\end{array}\right]
$$

According to [9], the infinitesimal operator $\mathcal{A}$ for diffusion process (9) acts on the function $g(t, \mathbf{X})$ from the class $C^{2}\left(\Re^{6}\right)$, where $g=g\left(t, r_{11}, r_{21}, r_{31}, r_{12}, r_{22}, r_{32}\right)$ and $\mathbf{R}_{j}=\left[r_{1 j}, r_{2 j}, r_{3 j}\right]^{\mathrm{T}}=\left[x_{j}, y_{j}, u_{x j}\right]^{\mathrm{T}}$, by the law

$$
\boldsymbol{A} g=\sum_{j=1}^{2} \sum_{i=1}^{3} B_{i j} \frac{\partial g}{\partial r_{i j}}+\frac{1}{2} \sum_{j=1}^{2}\left[\sum_{i=1}^{3} C_{i j} \frac{\partial^{2} g}{\partial r_{i j}^{2}}+C_{12 j} \frac{\partial^{2} g}{\partial r_{1 j} \partial r_{2 j}}\right]
$$

where all coefficients are uniquely determined

$$
\begin{aligned}
& B_{1 j}=v_{0 j} \cos \varphi_{j}-\alpha_{y j} \sin ^{2} \varphi_{j} r_{1 j}+\alpha_{y j} \sin \varphi_{j} \cos \varphi_{j} r_{2 j}+\cos \varphi_{j} r_{3 j} ; \\
& B_{2 j}=v_{0 j} \sin \varphi_{j}+\alpha_{y j} \sin \varphi_{j} \cos \varphi_{j} r_{1 j}-\alpha_{y j} \cos ^{2} \varphi_{j} r_{2 j}+\sin \varphi_{j} r_{3 j} ; \\
& B_{3 j}=-\alpha_{x j} r_{3 j} ; \quad C_{1 j}=\sigma_{y j}^{2} \sin ^{2} \varphi_{j} ; C_{2 j}=\sigma_{y j}^{2} \cos ^{2} \varphi_{j} ; \\
& C_{3 j}=\sigma_{x j}^{2} ; \quad C_{12 j}=-2 \sigma_{y j}^{2} \sin \varphi_{j} \cos \varphi_{j} .
\end{aligned}
$$

\subsection{Conditions of conflict detection}

Consider the conditions of conflict appearance. Suppose that two aircraft collide when their relative distance is less than or equal to safe distance $d$.

Denote by $q$ the probability of collision at time interval $[0, T]$

$$
q=P\{\exists t \in[0, T]:\|\mathbf{r}(t)\| \leq d\} .
$$

Introduce the function of conditional probability of collision at time interval $[T-t, T]$,

$$
\begin{aligned}
g(t, \mathbf{X}) & =P\{\exists \tau \in[T-t, T]:\|\mathbf{r}(\tau)\| \leq \\
& \leq d \mid \mathbf{X}(T-t)=\mathbf{X}\} .
\end{aligned}
$$

According to (6), collision occurs when $\mathbf{X}(t)$ hits the domain

$$
\mathbf{K}=\left\{\mathbf{X} \in \mathfrak{R}^{6}:\left\|\mathbf{r}_{0}+\mathbf{P}_{12}\left(\mathbf{R}_{2}-\mathbf{R}_{1}\right)\right\| \leq d\right\},
$$

where $\mathbf{X}=\left[\begin{array}{l}\mathbf{R}_{1} \\ \mathbf{R}_{2}\end{array}\right]$ and $\mathbf{P}_{12}=\left[\begin{array}{lll}1 & 0 & 0 \\ 0 & 1 & 0\end{array}\right]$

\subsection{Equation of conflict probability}

The problem of revealing aircraft conflict (13) is similar to the well-known problem of achieving boundaries by a multidimensional Markovian process for which a partial differential equation of parabolic type is defined in a general form [8].

According to L. Pontryagin, the function $g(t, \mathbf{X})$ (12) for infinitesimal operator (10) satisfies the parabolic partial differential equation $[8,4]$

$$
\frac{\partial g(t, \mathbf{X})}{\partial t}=\boldsymbol{A} g(t, \mathbf{X}), \quad t \in(0, T], \mathbf{X} \in \Re^{6} \backslash \mathbf{K}
$$

with initial and boundary conditions

$$
\begin{array}{ll}
g(t, \mathbf{X})=1, & \mathbf{X} \in \partial \mathbf{K}, \quad t \in(0, T], \\
g(0, \mathbf{X})=0, & \mathbf{X} \in \mathfrak{R}^{6} \backslash \mathbf{K} .
\end{array}
$$

The function $g$ has been defined and is continuous on the following set

$$
\begin{aligned}
D= & \left\{(t, \mathbf{X}): t \in(0, T], \mathbf{X} \in\left(\Re^{6} \backslash \mathbf{K}\right) \bigcup \partial \mathbf{K},\right. \\
& \text { or } \left.t=0, \mathbf{X} \in \mathfrak{R}^{6} \backslash \mathbf{K}\right\} .
\end{aligned}
$$

The solution of equation (14) yields the probability that the process achieves the boundary. The probability of collision (11) at time interval $[0, T]$ equals

$$
q=g(T, \mathbf{X}(0)), \quad \mathbf{X}(0) \in \Re^{6} \backslash \mathbf{K} .
$$

\section{Results of numerical solution}

Conflict probability equation (14) was solved for some conditions by the numerical method.

It was considered that two aircraft were flying straight at the same altitude along parallel paths and were approaching each other. For the OU process of deviation from the route line was taken $\alpha_{y}=0.01 s^{-1}$ and $\sigma_{y}=100 \mathrm{~m}$. The safe separation distance was considered $d=1000 \mathrm{~m}$. The steps of difference along coordinates $x$ and $y$ were taken to equal one hundred meters.

The results of the numerical solution are shown in figure 3, where it is shown how the probability of potential conflict depends on the relative position of the aircraft for different prediction time $T_{p}$, correspondingly 5 seconds (Fig 3, a), 10 seconds (Fig 3, b), and 20 seconds (Fig 3,c). 
The results are treated in the following way. The point assigned on the grid fixes the initial relative position of the two aircraft. The intersection of the perpendicular set up in this point with the surface representing the conflict equation solution gives the value of the probability of potential conflict for a specific prediction time.

It is seen that the solution is highly sensitive to relative aircraft position and prediction time for rather close aircraft approaching each other.
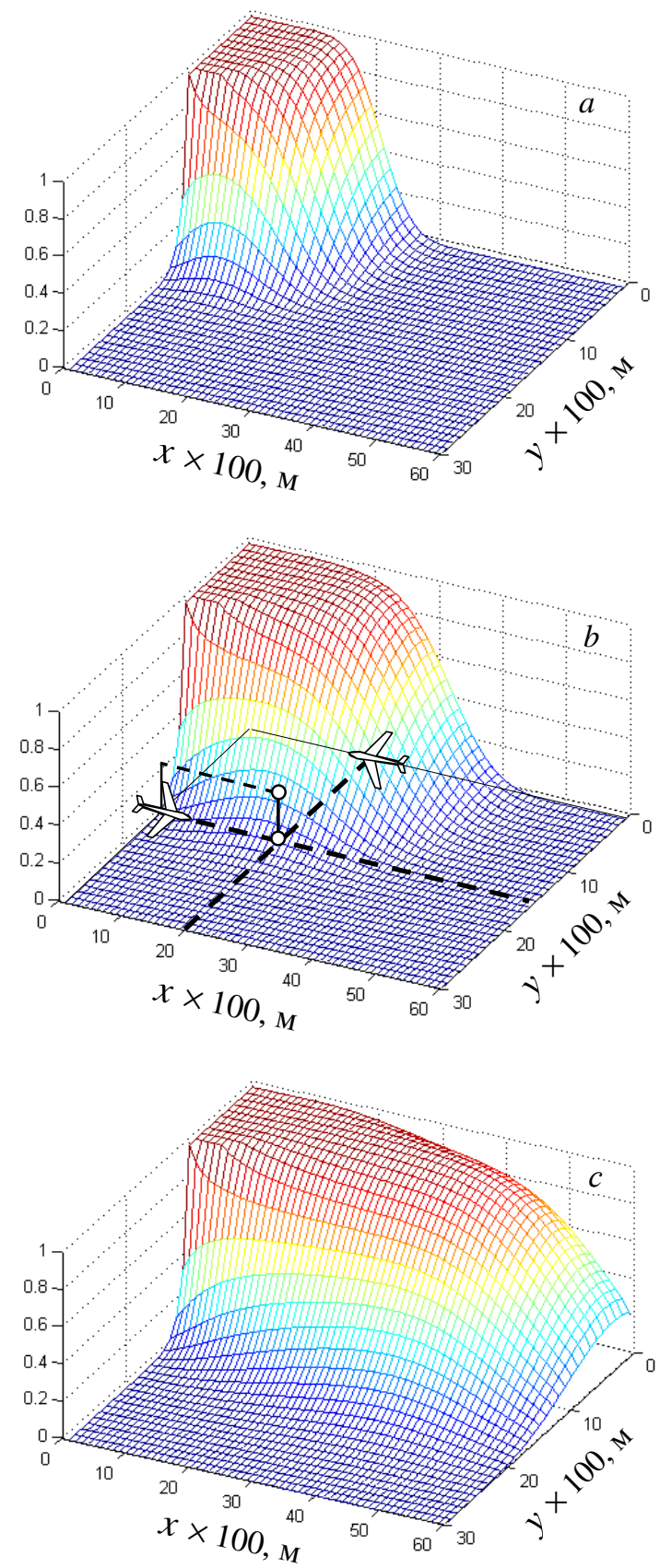

Fig 3. Conflict probability dependent on initial relative aircraft position for prescribed prediction time $T_{p}: a-T_{p}=5 \mathrm{~s} ; b$ $-T_{p}=10 \mathrm{~s} ; c-T_{p}=20 \mathrm{~s}$

\section{Conclusions}

The generalized stochastic conflict probability evaluation method is developed. In this method, taking into account the stochastic nature derives the conflict probability equation and time correlation of deviation from planned and controlled trajectory under condition of Cooperative ATM, when intended trajectories are known.

The mathematical formulation of the problem in a general case for aircraft flying at the same altitude and the procedure of evaluation are presented. The conflict probability equation is described as a multi-dimensional parabolic partial differential equation by using a differential (infinitesimal) operator of stochastic process of relative aircraft movement. All coefficients of the differential operator for a practical solution are derived.

Stabilization of the intended trajectory parameters by a flight management system is taken into account. To derive a mathematical model of deviations from a prescribed flight path, the Ornstein-Ulenbeck random process is used.

For some conditions, the numerical solution of the conflict probability equation is obtained and illustrated graphically. The solution is highly sensitive even at very close approach of aircraft-at a distance up to several hundreds meters. Thus, the presented generalized method permits one to estimate both conflict probability and collision risk with high reliability.

\section{References}

1. BABAK, V., KHARCHENKO, V., VASYLYEV, V. Methods of conflict probability estimation and decision making for air traffic management. Aviation. Vilnius: Technika, 2006, vol. 10, no. 1, p. 3-9.

2. BAKKER, GJ., KREMER, HJ., BLOM, HAP. Probabilistic approaches toward conflict prediction. In Air transportation systems engineering, AIAA, 2001, p. 677-694.

3. BLOM, HAP., BAKKER, GJ., BLANKER, PJG. et al. Accident risk assessment for advanced ATM. In Air transportation systems engineering. Edited by GL. Donohue and AG. Zellweger. 2001, p. 463-480.

4. KHARCHENKO, VP., KUKUSH, AG., VASYLYEV, VN. A generalized stochastic method of conflict performance evaluation in controlled air traffic. Kibernetica i sistemny analiz, 2005, no. 3, p. 81-93. In Russian.

5. Medium term concepts of operations: Co-operative air traffic management. In A European commission 6th framework project, 2005, p. 23.

6. System performance characteristics of centralized and decentralized air traffic separation strategies. USA /Europe Air Traffic Management R\&D Seminar, Santa Fe. 2001. Edited by J. Krozel, M. Peters, KD. Bilimoria, C. Lee and JSB. Mitchellin. http://atm2001.eurocontrol.fr/s1185.

7. Stochastic conflict detection model revisited. AIAA Guidance, navigation, and control conference. Denver, CO. Aug. 2000 Edited by K. Blin, M. Akian, F. Bonnans, E. Hoffman, C. Martini, KA. Zenghal. 
In www.eurocontrol.int/eec/public/standard_page/ SSP_2000_report_3.html

8. TIKHONOV, VI., MIRONOV, MA. Markovian processes. Moscow: Sov. Radio, 1977, p. 488. In Russian.

9. Towards cooperative ATS: The COOPATS concept. In European air traffic management programme, Eurocontrol, 2001, p. 54. 